\title{
ON THE HARDNESS OF APPROXIMATING MULTICUT AND SPARSEST-CUT
}

\author{
Shuchi Chawla, Robert Krauthgamer, Ravi Kumar, \\ YuVAl RABANi, AND D. SivaKumaR
}

\begin{abstract}
We show that the Multicut, Sparsest-Cut, and Min$2 \mathrm{CNF} \equiv$ DELETION problems are NP-hard to approximate within every constant factor, assuming the Unique Games Conjecture of Khot (2002). A quantitatively stronger version of the conjecture implies an inapproximability factor of $\Omega(\sqrt{\log \log n})$.

Keywords. Multicut, sparsest-cut, unique games conjecture, Fourier analysis.

Subject classification. 68Q17.
\end{abstract}

\section{Introduction}

In the MuLticut problem the input is an undirected graph $G=(V, E)$ on $n=|V|$ vertices together with $k$ pairs of vertices $\left\{s_{i}, t_{i}\right\}_{i=1}^{k}$, called demand pairs, and the goal is to find a minimum-size subset of the edges $M \subseteq E$ whose removal disconnects all the demand pairs, i.e., in the subgraph $(V, E \backslash M)$ every $s_{i}$ is disconnected from its corresponding vertex $t_{i}$. In the weighted version of this problem, the input also specifies a positive cost $c(e)$ for each edge $e \in E$ and the goal is to find a subset of the edges $M \subseteq E$ whose total cost $c(M)=\sum_{e \in M} c(e)$ is minimal. This problem is known to be APX-hard (Dahlhaus et al. 1994).

We prove that if the Unique Games Conjecture of Khot (2002) is true, then for every constant $L>0$ it is NP-hard to approximate MULTicuT within factor $L$. If a quantitatively stronger version of the conjecture is true, then Multicut is NP-hard to approximate within a factor of $\Omega(\sqrt{\log \log n})$.

Our methods also yield similar bounds for the SPARSEST-Cut problem and the Min-2CNF $\equiv$ DeLETION problem. The Sparsest-Cut problem has the same input as Multicut, but the goal is to find a subset of the edges $M \subseteq E$ that minimizes the ratio of $|M|$ (in the weighted version, the total cost of $M$ ) to the number of demand pairs that are disconnected in 
$(V, E \backslash M) .{ }^{1} \quad$ Since Sparsest-Cut is not known to be APX-hard, our result gives the first indication that this problem might be hard to approximate. In the Min-2CNF $\equiv$ DELETION problem the input is a weighted set of clauses on $n$ variables, each clause of the form $(x \equiv y)$, where $x$ and $y$ are literals, and the goal is to find a Boolean assignment to the variables minimizing the total weight of unsatisfied clauses. ${ }^{2}$ Our results immediately extend also to the Correlation Clustering problem (Bansal et al. 2004; Charikar et al. 2003; Demaine \& Immorlica 2003; Emanuel \& Fiat 2003) of minimizing disagreements in a weighted graph, since the approximability of this problem is known to be equivalent to within constant factors to that of MULTICUT in weighted graphs (Charikar et al. 2003; Emanuel \& Fiat 2003).

1.1. Known results on Multicut, Sparsest-Cut, and Min-2CNF $\equiv$ Deletion. Multicut and Sparsest-Cut are fundamental combinatorial optimization problems, with connections to multicommodity flow, edge expansion, and metric embeddings. Both problems can be approximated to within $O(\log k)$ factors through linear programming (LP) relaxations (Aumann \& Rabani 1998; Garg et al. 1996; Leighton \& Rao 1988; Linial et al. 1995). These bounds match, up to constant factors, the lower bounds on the integrality gaps of the corresponding relaxations (Garg et al. 1996; Leighton \& Rao 1988). Min$2 \mathrm{CNF} \equiv$ DeLETION can also be approximated to within an $O(\log n)$ factor, as implied by the results of Klein et al. (Klein et al. 1990) (see also Vazirani 2001, Section 20.4), who give an approximation-preserving reduction from this problem to Multicut. Recently, improved approximation algorithms for the SPARSEST-CUT problem have been developed using a semidefinite programming (SDP) relaxation (Arora et al. 2005, 2004; Chawla et al. 2005a). This started with the ground-breaking $O(\sqrt{\log n})$-approximation of Arora, Rao \& Vazirani (2004) for the uniform demands case, and the best approximation factor currently known for general demands is $O(\sqrt{\log k} \log \log k$ ) (Arora et al. 2005). Agarwal et al. (2005) extended these techniques to obtain an $O(\sqrt{\log n})$ approximation for the Min-2CNF $\equiv$ DELETION problem. The obvious modification of the semidefinite program used for SPARSEST-CuT to solve Multicut was recently shown to have an integrality ratio of $\Omega(\log k)$ (Agarwal et al. 2005, Section 6), which matches, up to constant factors, the approximation factor and

\footnotetext{
${ }^{1}$ In general, the demand pairs may have positive weights (demands), but for our purpose of inapproximability results, it clearly suffices to consider the more restricted definition above. Our hardness results for SPARSEST-CuT do not apply to the special case of uniform-demand, in which every pair of vertices forms a demand pair.

${ }^{2}$ Note that the constraints in Min-2CNF $\equiv$ DELETION are restricted to equality (and effectively non-equality) constraints.
} 
integrality gap of previously analyzed linear programming relaxations for this problem.

On the hardness side, it is known that Multicut is APX-hard (Dahlhaus et al. 1994), i.e., there exists a constant $c>1$ such that it is NP-hard to approximate MULTiCUT to within a factor smaller than $c$. It should be noted that this hardness of approximation holds even for $k=3$, and that the value of $c$ is not specified therein, but it is certainly smaller than 2. The Min$2 \mathrm{CNF} \equiv$ DELETION problem is also known to be APX-hard, as follows, e.g., from the hardness of approximating linear equations modulo 2 (Håstad 2001).

Assuming the Unique Games Conjecture, Khot (2002, Theorem 3) essentially obtained an arbitrarily large constant-factor hardness of approximation for Min-2CNF $\equiv$ Deletion, and this implies, using the aforementioned reduction of Klein et al. (1990), a similar hardness factor for Multicut. These results are not explicitly noted in Khot (2002), and are weaker than our results in several respects. First, our quantitative bounds are better; thus if a stronger, yet almost as plausible, version of this conjecture is true, then our lower bound on the approximation factor improves to $L=\Omega(\sqrt{\log \log n})$, compared with the roughly $\Omega\left((\log \log n)^{1 / 4}\right)$ hardness that can be inferred from Khot (2002); this can be viewed as progress towards proving tight inapproximability results for Multicut. Second, by qualitatively strengthening our Multicut result to a bicriteria version of the problem, we extend our hardness results to the SPARSEST-CUT problem. It is unclear whether Khot's reduction similarly leads to a hardness result for SpARSEST-CUT. Finally, our proof is simpler (both the reduction and its analysis), and makes direct connections to cuts (in a hypercube), and thus may prove useful in further investigation of such questions.

For Sparsest-Cut, no hardness of approximation result was previously known. Independently of our work, Khot \& Vishnoi (2005) have recently used a different construction to show an arbitrarily large constant factor hardness for Sparsest-Cut assuming the Unique Games Conjecture. Their hardness factor could, in principle, be pushed to $(\log \log n)^{1 / 4-o(1)}$, assuming a stronger quantitative version of the conjecture. Additionally, they prove a lower bound of $(\log \log n)^{1 / 4-o(1)}$ on the integrality ratio of the semidefinite programming relaxations used in the recent approximation algorithms for SpARSEST-CUT. This last lower bound was further improved to $\Omega(\log \log n)$ in Krauthgamer \& Rabani (2006), using in part ideas from the current paper.

1.2. The Unique Games Conjecture. A unique 2-prover game is the following problem. The input is a bipartite graph $G_{Q}=\left(Q, E_{Q}\right)$, where each side $p=1,2$ contains $n=|Q| / 2$ vertices denoted $q_{1}^{p}, \ldots, q_{n}^{p}$, and represents $n$ 
possible questions to prover $p$. In addition, the input contains for each edge $\left(q_{i}^{1}, q_{j}^{2}\right) \in E_{Q}$ a non-negative weight $w\left(q_{i}^{1}, q_{j}^{2}\right)$, abbreviated $w_{i j}$. These edges will be called question edges, to distinguish them from edges in the MulticuT instance. Each question to a prover is associated with a set of $d$ distinct answers, denoted by $[d]=\{1, \ldots, d\}$. The input also contains, for every question edge $\left(q_{i}^{1}, q_{j}^{2}\right) \in E_{Q}$, a bijection $b_{i j}:[d] \rightarrow[d]$, which maps every answer for question $q_{i}^{1}$ to a distinct answer for question $q_{j}^{2}$.

A solution $A$ to the 2-prover game consists of an answer $A_{i}^{p} \in[d]$ for each question $q_{i}^{p}$ (i.e., a sequence $\left\{A_{i}^{p}\right\}$ over all $p \in[2]$ and $i \in[n]$ ). The solution is said to satisfy a question edge $\left(q_{i}^{1}, q_{j}^{2}\right) \in E_{Q}$ if the answers $A_{i}^{1}$ and $A_{j}^{2}$ agree under their bijection, i.e., $A_{j}^{2}=b_{i j}\left(A_{i}^{1}\right)$. We assume that the total weight of all the edges in $E_{Q}$ is 1 (by normalization). The value of a solution is the total weight of all the edges satisfied by the solution. The value of the game is the maximum value achievable by any solution to the game.

Conjecture 1.1 (Unique Games Conjecture, Khot 2002). For every fixed $\eta, \delta>0$ there exists $d=d(\eta, \delta)$ such that it is NP-hard to determine whether a unique 2-prover game with answer set size $d$ has value at least $1-\eta$ or at most $\delta$.

We will also consider stronger versions of the Unique Games Conjecture in which $\eta, \delta$, and $d$ are functions of $n$. Specifically, we will consider versions with $\eta \leq O(1 / \sqrt{\log \log n}), \delta \leq 1 /(\log n)^{\Omega(\eta)}$, and $d=d(\eta, \delta) \leq O(\log n)$. The reason for the latter upper bound is that our construction size is exponential in $d$.

Plausibility of the conjecture and stronger versions of it. The Unique Games Conjecture has been used to show optimal inapproximability results for Vertex Cover (Khot \& Regev 2003) and MAX-Cut (Khot et al. 2004; Mossel et al. 2005). Proving the conjecture using current techniques appears to be quite hard. In particular, the asserted NP-hardness is much stronger than what we can obtain via standard constructions using the PCP theorem (Arora et al. 1998; Arora \& Safra 1998) and the parallel repetition theorem (Raz 1998), two deep results in computational complexity.

Although the conjecture seems difficult to prove in general, some special cases are well-understood. In particular, if at all the Unique Games Conjecture is true (and assuming $\mathrm{P} \neq \mathrm{NP}$ ), then necessarily $d \geq \max \left\{1 / \eta^{1 / 10}, 1 / \delta\right\}$. This follows from a rounding procedure for a semidefinite programming relaxation presented in Khot (2002). On the other hand, Feige \& Reichman (2004) showed that for every constant $L>0$ there exists a constant $\delta>0$ such that it is NPhard to distinguish whether a unique 2-prover game (with $d=d(L, \delta)$ ) has 
value at least $L \delta$ or at most $\delta$; this result falls short of the Unique Games Conjecture in that $L \delta$ is bounded away from 1 .

Stronger versions of the conjecture in which $d, \eta$, and $\delta$ are functions of $n$ have also been considered. Trevisan (2005) and Gupta \& Talwar (2006) recently developed approximation algorithms for solving instances of Unique Games where $\eta(n)$ is a sufficiently small function of $n$ (based on an SDP and an LP relaxation respectively, different from the one used in Khot 2002). These algorithms imply that a stronger version of the Unique Games Conjecture can only be true if $\eta(n)=\Omega(1 / \log n)$ (assuming $\mathrm{P} \neq \mathrm{NP}$ ).

Very recently, Charikar et al. (2006) improved upon the rounding algorithm of Khot (2002) to obtain better approximation algorithms for Unique Games. Their results imply that for Unique Games Conjecture to be true, we must have $\eta \geq \Omega(1 / \log d)$ and $\delta \geq d^{-\Omega(\eta)}$. There are two pieces of evidence suggesting that, if the Unique Games Conjecture is true at all, then these bounds might be tight, i.e. Unique Games might in fact be hard for suitable $d=\Theta(\log n)$, and every $\delta \geq d^{-\Omega(\eta)}$ and $1 / \log d \ll d \eta<1 / 2$. First, these bounds nearly match the integrality gap of Khot \& Vishnoi (2005) for the natural SDP relaxation of Unique Games. Second, a recent version of Khot et al. (2004) shows (see Corollaries 13 and 15 of its ECCC version) that the standard Unique Games Conjecture implies hardness of Unique Games instances with the above tradeoff between parameters (namely, $\delta=d^{-\eta /(2-\eta)}$ ), for every $\eta>0$ that is fixed (independent of $n$ ) and $d$ larger than some $d_{0}(\eta)$ that is unspecified.

Our $\Omega(\sqrt{\log \log n})$ hardness result (see Corollary 1.4 below) requires $\eta \leq$ $O(1 / \sqrt{\log \log n}), \delta \leq 1 /(\log n)^{\Omega(\eta)}$, and $d=d(\eta, \delta) \leq O(\log n)$, which is still plausible by these results. Note that this setting of parameters is similar to, but stronger than, those in the aforementioned result of Khot et al. (2004), because the parameters do depend on $n$.

1.3. Our results. We prove the following hardness of approximation for Multicut, Sparsest-Cut, and Min-2CNF $\equiv$ Deletion based on Khot's Unique Games Conjecture.

TheOrem 1.2. There exist constants $c_{1}, c_{2}>0$ such that the following holds. Suppose that for $\eta=\eta(n), \delta=\delta(n)$, and $d=d(\eta, \delta) \leq O(\log n)$, it is NPhard to determine whether a unique 2-prover game with $|Q|=2 n$ vertices and answer set size $d$ has value at least $1-\eta(n)$ or at most $\delta(n)$. Then it is NP-hard to approximate Multicut, Sparsest-Cut, and Min-2CNF $\equiv$ Deletion to within factor

$$
L(n)=c_{1} \min \left\{\frac{1}{\eta\left(n^{c_{2}}\right)}, \log \frac{1}{\delta\left(n^{c_{2}}\right)}\right\} .
$$


This theorem immediately implies the following two specific hardness results.

Corollary 1.3. The Unique Games Conjecture implies that, for every constant $L>0$, it is NP-hard to approximate Multicut, Sparsest-Cut, and Min-2CNF $\equiv$ DELETION to within factor $L$.

Corollary 1.4. A stronger version of the Unique Games Conjecture in which $\eta \leq O(1 / \sqrt{\log \log n}), \delta \leq 1 /(\log n)^{\Omega(\eta)}$, and $d=d(\eta, \delta) \leq O(\log n)$ implies that for some fixed $c>0$, it is NP-hard to approximate Multicut, Sparsest-Cut, and Min-2CNF $\equiv$ DELETION to within factor $c \sqrt{\log \log n}$.

For Sparsest-Cut, our hardness results hold only for the search version (in which the algorithm needs to produce a cutset, i.e., a subset of edges, and not only its value), since our proof employs a Cook reduction. As noted before, a similar (but slightly weaker) bound is proved independently in Khot \& Vishnoi (2005).

Remark. The conference version of our paper (Chawla et al. 2005b) presented a different bound than the one of Theorem 1.2, and argued that an $\Omega(\log \log n)$ hardness result follows from a certain strong version of the Unique Games Conjecture, that has since been proven false by Charikar et al. (2006). Consequently, the current version of the paper is different in two respects. First, we use a different strong version of the Unique Games Conjecture that is still plausibly true by known evidence (and is in fact weaker than the one used in Chawla et al. 2005b). Second, the bound presented here in Theorem 1.2 is better, since in Chawla et al. (2005b) the dependence of $L(n)$ on $\eta$ was $c_{1}\left(\log \left(1 / \eta\left(n^{c_{2}}\right)\right)\right)$. Interestingly, the two versions employ exactly the same reduction, but the current analysis is different (and perhaps simpler), as it uses Friedgut's Junta Theorem (Friedgut 1998) rather than a theorem of Kahn et al. (1988). This improvement was also motivated, in part, by the integrality ratio of Khot \& Vishnoi (2005) for Unique Games, which suggests a significant asymmetry between $\eta(n)$ and $\delta(n)$.

\subsection{Preliminaries}

Regular unique games. A unique 2-prover game is called regular if the total weight of question edges incident at any single vertex is the same, i.e., $1 / n$, for every vertex in $Q$. We now show that we can assume without loss of generality that the graph in the Unique Games Conjecture is regular. For simplicity, we state this only for fixed $\eta$ and $\delta$. A similar result holds when they depend on $n$, 
because we increase the input size by no more than a polynomial factor, and increase $\eta$ and $\delta$ by no more than a constant factor.

Lemma 1.5. The Unique Games Conjecture implies that for every fixed $\eta, \delta$ $>0$, there exists $d=d(\eta, \delta)$ such that it is NP-hard to decide if a regular unique 2 -prover game with $n$ vertices and $d$ answers for each vertex has value at least $1-\eta$ or at most $\delta$.

Proof. Given a unique 2-prover game $Q$, we describe how to convert it to a regular game while (nearly) preserving its completeness and soundness. Let the maximum weight of any edge in $Q$ be $w_{\max }=\max _{e} w_{e}$. Let $r=\max \{1 / \eta, 1\}$. First we remove all edges of weight less than $\left(1 / 2 n^{2} r\right) w_{\max }$ from the graph, and renormalize the weights of the remaining edges so that they still sum to 1 . Note that the optimal solution to the game has value at least $w_{\max }$, and this edge-removal step reduces the value of any solution by at most $(1 / 2 r) w_{\max }$. So any solution of value $1-\eta$ in the original game now has value at least $1-\eta-1 / 2 r>1-3 \eta / 2$, and any solution of value at most $\delta$ in the original game now has value at most $\delta(1+1 / 2 r)<3 \delta / 2$.

Next, let $w_{\text {min }}$ be the minimum weight of any edge. We round down weights of all edges to the nearest multiple of $t=(1 / 2 r) w_{\min }$, and renormalize the weights so that they sum to 1 . As before, we only remove a $1 / 2 r$ fraction of the total weight and renormalize by a factor of at most $1+1 / 2 r$. Therefore, this changes the soundness and completeness parameters by at most an additive $1 / 2 r$, as before. Overall, the parameters change by a factor of at most 2 .

Now we convert the pre-processed game $Q$ to a regular graph $Q^{\prime}$ as follows. For each prover $p \in\{1,2\}$ and question $q_{i}^{p}$, form $W(p, i) / t$ vertices $q_{i}^{p}(1), \ldots, q_{i}^{p}(W(p, i) / t)$, where $W(p, i)$ is the total weight of all the edges incident on $q_{i}^{p}$. For every pair of vertices $\left(q_{i}^{1}, q_{j}^{2}\right)$, connected by an edge $e$ in $Q$, we form an edge between $q_{i}^{1}(x)$ and $q_{j}^{2}(y)$, for all possible values of $x$ and $y$, with weight $w_{e}(t / W(1, i))(t / W(2, j))$. The bijection on the edge $\left(q_{i}^{1}(x), q_{j}^{2}(y)\right)$ is set to be the same as the original bijection $b_{i j}$ on the edge $\left(q_{i}^{1}, q_{j}^{2}\right)$.

Note that the total weight of all the edges remains the same as before. Each new vertex $q_{i}^{1}(x)$ has total weight

$$
\sum_{e} w_{e} \frac{t}{W(1, i)} \frac{t}{W(2, j)} \frac{W(2, j)}{t}=t
$$

where the sum is over all edges $e$ incident on $q_{i}^{1}$. The same holds for any new vertex $q_{j}^{2}(y)$. Therefore, the graph is regular. Furthermore, the number of vertices increases by a factor of at most $4 n^{2} r^{2}$. 
It only remains to show that the soundness and completeness parameters are preserved. To see this, note that any solution on the original graph $Q$ can be transformed to a solution of the same value on $Q^{\prime}$, by picking the same answer for every vertex $q_{i}^{p}(x)$ in $Q^{\prime}$ as the answer picked for $q_{i}^{p}$ in $Q$. Likewise, consider a solution in $Q^{\prime}$. Note that for fixed $p$ and $i$, all questions $q_{i}^{p}(x)$ are connected to identical sets of vertices with identical bijections. Thus, we can replace answers to all these questions by the answer to the question $q_{i}^{p}(x)$ that has the most satisfied edges incident on it, thereby increasing the value of the solution. Now, the solution in $Q$ that picks the same answer for $q_{i}^{p}$ as the answer for $q_{i}^{p}(x)$ in $Q^{\prime}$ has the same weight as the new solution in $Q^{\prime}$ which is at least as much as the value of the original solution in $Q^{\prime}$.

Thus for every solution in $Q$ (after pre-processing), there is a solution of the same or larger weight in $Q^{\prime}$ and vice versa. This proves that the two games have exactly the same soundness and completeness parameters.

The relationship between $d, \eta, \delta$ and $n$ is important for our purposes. We thus point out that this argument changes the parameters $\delta$ and $\eta$ by at most a factor of 2 , increases the size of the instance by at most a polynomial factor in $n, 1 / \eta$ and $1 / \delta$, and does not change $d$. This is acceptable in the setting of Theorem 1.2 as well as Corollary 1.4 - the requirements on $d, \eta$ and $\delta$ as functions of $n$ are maintained and only the unspecified constants therein are affected.

Bicriteria Multicut. Our hardness of approximation proof for SpARSESTCut relies on a generalization of Multicut, where the solution $M$ is required to cut only a certain fraction of the demand pairs. For a given graph $G=(V, E)$, a subset of the edges $M \subseteq E$ will be called a cutset of the graph. A cutset whose removal disconnects all the demand pairs is a multicut.

An algorithm is called an $(\alpha, \beta)$-bicriteria approximation for Multicut if, for every input instance, the algorithm outputs a cutset $M$ that disconnects at least an $\alpha$ fraction of the demands and has cost at most $\beta$ times that of the optimum multicut. In other words, if $M^{*}$ is a least cost cutset that disconnects all the $k$ demand pairs, then $M$ disconnects at least $\alpha k$ demand pairs and $c(M) \leq \beta c\left(M^{*}\right)$.

Hypercubes, dimension cuts, and antipodal vertices. As usual, the $d$-dimensional hypercube (for short, a $d$-cube) is the graph $C=\left(V_{C}, E_{C}\right)$ with the vertex set $V_{C}=\{0,1\}^{d}$, and an edge $(u, v) \in E_{C}$ for any two vertices $u, v \in\{0,1\}^{d}$ that differ in exactly one dimension (coordinate). An edge $(u, v)$ 
is called a dimension- $a$ edge, for $a \in[d]$, if $u$ and $v$ differ in dimension $a$, i.e., $u \oplus v=1_{a}$ where $1_{a}$ is a unit vector along dimension $a$. The set of all the dimension- $a$ edges in the hypercube is called the dimension- $a$ cut in the hypercube; a dimension cut is a dimension- $a$ cut for some dimension $a$. The antipode of a vertex $u$ is the (unique) vertex $\bar{u}$ all of whose coordinates are different from those of $u$, i.e., $\bar{u}=u \oplus \mathbf{1}$ where $\mathbf{1}$ is the vector with 1 in every coordinate. Notice that $v$ is the antipode of $u$ if and only if $u$ is the antipode of $v$; thus, $\{u, \bar{u}\}$ form an antipodal pair. The following simple fact will be key in our proof.

FACT 1.6. In every hypercube, a single dimension cut disconnects every antipodal pair.

Organization. In Section 2 we prove the part of Theorem 1.2 regarding the MuLTiCuT problem; our proof will actually hold for bicriteria approximation for Multicut. We will then show in Section 3 that this stronger result yields a similar hardness of approximation for SpARSEST-CuT. Finally, in Section 4, we modify the reduction to obtain a hardness of approximation for MIN-2CNF $\equiv$ DELETION.

\section{Hardness of bicriteria approximation for Multicut}

In this section we prove the part of Theorem 1.2 regarding the MulTiCuT problem, namely, that the Unique Games Conjecture implies that it is NPhard to approximate MUlTiCUT within a certain factor $L$. Our proof will actually show a stronger result - for every $\alpha \geq 7 / 8$ it is NP-hard to distinguish between whether there is a multicut of cost less than $n 2^{d+1}$ (the YES instance) or whether every cutset that disconnects at least $\alpha k$ demand pairs has cost at least $n 2^{d+1} L$ (the NO instance). This implies that it is NP-hard to obtain an $(\alpha, L)$-bicriteria approximation for Multicut.

We start by describing a reduction from unique 2-prover game to Multicut (Section 2.1), and then proceed to analyze the YES instance (Section 2.2) and the NO instance (Sections 2.3 and 2.4). Finally, we discuss the gap that is created for a bicriteria approximation of Multicut (Section 2.5).

2.1. The reduction. Given a regular unique 2-prover game instance $G_{Q}=$ $\left(Q, E_{Q}\right)$ with $n=|Q| / 2$ and the corresponding edge weights $w_{i j}$ and bijections $b_{i j}:[d] \rightarrow[d]$, we construct a Multicut instance $G=(V, E)$ with demand pairs, as follows. For every vertex (i.e., question) $q_{i}^{p} \in Q$, construct a $d$ dimensional hypercube $C_{j}^{p}$; the dimensions in this cube correspond to answers 
for the question $q_{j}^{p}$. For each of the $2 n$ hypercubes, we let the edges inside the hypercube have cost 1 , and call them hypercube edges. ${ }^{3}$

For each question edge $\left(q_{i}^{1}, q_{j}^{2}\right) \in E_{Q}$, we extend $b_{i j}$ (in the obvious way) to a bijection from the vertices of $C_{i}^{1}$ to the vertices of $C_{j}^{2}$, and denote the resulting bijection by $b_{i j}^{\prime}:\{0,1\}^{d} \rightarrow\{0,1\}^{d}$. Formally, for every $u \in\{0,1\}^{d}$ (vertex in $C_{i}^{1}$ ) and every $a \in[d]$, the $a$-th coordinate of $b_{i j}^{\prime}(u)$ is given by $\left(b_{i j}^{\prime}(u)\right)_{a}=u_{b_{i j}^{-1}(a)}$. Then we connect every vertex $u \in C_{i}^{1}$ to the corresponding vertex $b_{i j}^{\prime}(u) \in C_{j}^{2}$ using an edge of cost $w_{i j} \Lambda$, where $\Lambda=n / \eta$ is a scaling factor. These edges are called cross edges.

Denote the resulting graph by $G=(V, E)$. Notice that $V$ is simply the union of the vertex sets of the hypercubes $C_{i}^{p}$, for all $p \in[2]$ and $i \in[n]$, and that the edge set $E$ contains two types of edges, hypercube edges and cross edges.

To complete the reduction, it remains to define the demand pairs. For a vertex $u \in V$, the antipode of $u$ in $G$, denoted $\bar{u}$, is defined to be the vertex antipodal to $u$ in the hypercube $C_{i}^{p}$ that contains $u$. The set $D$ of demand pairs then contains every pair of antipodal vertices in $G$, and since the graph contains $2 n$ hypercubes, $k=|D|=n 2^{d}$. Note that every vertex of $G$ belongs to exactly one demand pair.

\subsection{The YES instance}

Lemma 2.1. If there is a solution $A$ for the unique 2-prover game $G_{Q}$ such that the total weight of the satisfied questions is at least $1-\eta$, then the resulting Multicut instance $G$ contains a multicut $M \subseteq E$ such that $c(M) \leq 2^{d+1} n$.

Proof. Let $A$ be such a solution for $G_{Q}$. Construct $M$ by taking the following edges. For every question $q_{i}^{p} \in Q$ and the corresponding answer $A_{i}^{p}$ (of prover $p$ ), take the dimension- $A_{i}^{p}$ cut in cube $C_{i}^{p}$. In addition, for every edge $\left(q_{i}^{1}, q_{j}^{2}\right) \in E_{Q}$ that the solution $A$ does not satisfy, take all the cross edges between the corresponding cubes $C_{i}^{1}$ and $C_{j}^{2}$.

We first claim that removing $M$ from $G$ disconnects all the demand pairs. To see this, we define a Boolean function $f: V \rightarrow\{0,1\}$ on the graph vertices. For every cube $C_{i}^{p}$, consider the dimension- $A_{i}^{p}$ cut; it disconnects the cube into two connected components, one containing the all-zeros vector $\mathbf{0}$ and one containing the all-ones vector 1 . For every $v \in C_{i}^{p}$, let $f(v)=0$ if $v$ is in the

\footnotetext{
${ }^{3}$ This is a standard technique in PCP constructions for graph optimization problems. A hypercube can be interpreted as a "long code" (Bellare et al. 1998), and a dimension cut is the encoding of an answer in the 2-prover game.
} 
same component as $\mathbf{0}$, and $f(v)=1$ otherwise. This is exactly the $A_{i}^{p}$-th bit in $v$, i.e., $f(v)=v_{A_{i}^{p}}$. Now consider any demand pair $(v, \bar{v})$, and note that $f(v)=1-f(\bar{v})$. We will show below that every edge $(u, v) \notin M$ has the property $f(u)=f(v)$. This will clearly complete the proof of the claim.

Consider first a hypercube edge $(u, v)$ in $C_{i}^{p}$ that is not a dimension- $A_{i}^{p}$ edge. Then $f(u)=u_{A_{i}^{p}}=v_{A_{i}^{p}}=f(v)$, by the definition of $f$. Next consider a cross edge $(u, v) \notin M$. Then this edge lies between cubes $C_{i}^{1}$ and $C_{j}^{2}$ such that the question edge $\left(q_{i}^{1}, q_{j}^{2}\right)$ is satisfied by the unique 2-prover game solution $A$. Therefore, $b_{i j}\left(A_{i}^{1}\right)=A_{j}^{2}$ and $f(u)=u_{A_{i}^{1}}=v_{b_{i j}\left(A_{i}^{1}\right)}=v_{A_{j}^{2}}=f(v)$.

Finally, we bound the cost of the solution. Let $S$ be the set of question edges not satisfied by the solution $A$. The total cost of the multicut solution is thus

$$
c(M)=2 n \cdot 2^{d-1}+2^{d} \Lambda \sum_{\left(q_{i}^{1}, q_{j}^{2}\right) \in S} w_{i j} \leq 2^{d} n+2^{d} \Lambda \eta=2^{d+1} n .
$$

2.3. Hypercube cuts, Boolean functions, influences, and juntas. We will analyze the NO instance shortly, but first we set up some notation and present a few technical lemmas regarding cuts in hypercubes. In particular, we present Theorem 2.2, which will have a crucial role in what follows.

Recall that the dimensions of the hypercubes in the multicut instance correspond to answers to the 2-prover game. Therefore, we would like to determine which dimensions are the most significant participants in a cut on the cube, as follows. Let $C=\left(V_{C}, E_{C}\right)$ be a $d$-dimensional hypercube. It will be useful to represent cuts on the hypercube $C$ as functions $f: V_{C} \rightarrow \mathbb{Z}$. Such a function $f$ corresponds to a partition of $V_{C}$ into sets $\left\{f^{-1}(r): r \in f\left(V_{C}\right)\right\}$, which in turn corresponds to the cutset $\left\{(u, v) \in E_{C}: f(u) \neq f(v)\right\}$. Notice that $f$ can be described as a function on $d$ Boolean variables (corresponding to the dimensions of the hypercube), where the dimension $a \in[d]$ corresponds to the $a$-th variable. The influence of a dimension (variable) $a \in[d]$ on the function $f$, denoted $I_{a}^{f}$, is defined as the fraction of the dimension $a$-edges $(u, v) \in E_{C}$ for which $f(u) \neq f(v)$. In other words, $I_{a}^{f}=\operatorname{Pr}_{u \in V_{C}}\left[f(u) \neq f\left(u \oplus 1_{a}\right)\right]$ where $1_{a}$ is a unit vector along dimension $a$. The total influence (also called average sensitivity) of $f$ is $\sum_{a \in[d]} I_{a}^{f}$. We say that the function $f$ is a $k$-junta if there exists a subset $J \subseteq[d]$ with $|J| \leq k$ such that for every variable (dimension) $a \notin J$ and for every $u \in V_{C}$, we have $f(u)=f\left(u \oplus 1_{a}\right)$. In other words, $f$ depends on at most $k$ variables, and the remaining variables have zero influence. Two functions $f$ and $f^{\prime}$ are said to be $\varepsilon$-close if $\operatorname{Pr}_{u \in V_{C}}\left[f(u) \neq f^{\prime}(u)\right] \leq \varepsilon$.

An important special case is that of Boolean functions, i.e., $g: V_{C} \rightarrow$ $\{0,1\}$, which corresponds to a bipartition of $V_{C}$. The balance of a Boolean 
function $g$ is defined as $\min \left\{\left|g^{-1}(0)\right| /\left|V_{C}\right|,\left|g^{-1}(1)\right| /\left|V_{C}\right|\right\}$, i.e., the minimum of $\operatorname{Pr}_{u \in V_{C}}[g(u)=0]$ and $\operatorname{Pr}_{u \in V_{C}}[g(u)=1]$.

The next theorem, due to Friedgut (1998), asserts that every Boolean function of low total influence is close to a junta. We will later use it to determine a set of dimensions that are the most significant participants in a low-cost cutset.

Theorem 2.2 (Friedgut's (1998) Junta Theorem). Let $g$ be a Boolean function defined on a hypercube and fix $\varepsilon>0$. Then $g$ is $\varepsilon$-close to a Boolean function $h$ defined on the same cube and depending on only $2^{O(T / \varepsilon)}$ variables, where $T=\sum_{a \in[d]} I_{a}^{g}$ is the total influence of $g$.

\subsection{The NO instance}

Lemma 2.3. There exists $L=\Omega\left(\min \left\{\eta^{-1}, \log \delta^{-1}\right\}\right)$ such that if the MultiCUT instance $G$ has a cutset of cost at most $2^{d+1} n L$ whose removal disconnects an $\alpha \geq 7 / 8$ fraction of the demand pairs, then there is a solution $A$ for the unique 2-prover game $G_{Q}$ whose value is larger than $\delta$.

Proof. Let $L=c \min \{1 / \eta, \log (1 / \delta)\}$ for a constant $c>0$ to be determined later, and let $M \subseteq E$ be a cutset of cost $c(M) \leq 2^{d+1} n L$ whose removal disconnects an $\alpha \geq 7 / 8$ fraction of the demand pairs. Using $M$, we will construct for the unique 2-prover game $G_{Q}$ a randomized solution $A$ whose expected value is larger than $\delta$, thereby proving the existence of a solution of value larger than $\delta$. The randomized solution $A$, which is a strategy for the two provers, is defined as follows. Label each connected component of $G \backslash M$ as either 0 or 1 independently at random with equal probabilities, and define a Boolean function $f: V \rightarrow\{0,1\}$ by letting $f(v)$ be the label of the connected component of $v \in V$. Next, for each vertex (question) $q_{i}^{p} \in Q$ consider the restriction of $f$ to the hypercube $C_{i}^{p} \subset V$, denoted $f_{\mid C_{i}^{p}}$, and apply to it Theorem 2.2 (Friedgut's Junta Theorem) with $\varepsilon=1 / 60$, to obtain a subset of the variables (dimensions) $J_{i}^{p} \subseteq[d]$; the idea is that for many hypercubes we obtain $\left|J_{i}^{p}\right| \leq 2^{O(L / \varepsilon)}$. Finally, choose the answer $A_{i}^{p}$ uniformly at random from $J_{i}^{p}$, independently of all other events.

We proceed to analyze the expected value of this randomized solution $A$. Recall that the value of a solution is equal to the probability that, for a question edge $\left(q_{i}^{1}, q_{j}^{2}\right)$ chosen at random with probability proportional to its weight, we have $a_{j}^{2}=b_{i j}\left(a_{i}^{1}\right)$. Notice that although $q_{i}^{1}$ and $q_{j}^{2}$ are correlated, each one is uniformly distributed because $Q$ is regular. Without loss of generality, we assume that removing $M$ disconnects at least as many demand pairs inside the cubes $\left\{C_{l}^{1}\right\}_{l \in[n]}$ as inside the cubes $\left\{C_{l}^{2}\right\}_{l \in[n]}$. Now we claim that with a 
constant probability over the choice of a question edge, the cut $M$ has a low cost over edges incident on the two corresponding hypercubes, and disconnects many demand pairs in these two hypercubes. In other words, the quality of the cut locally is nearly as good as the quality of the cut globally. In particular, we upper bound the probability of the following four "bad" events (for a random question edge $\left.\left(q_{i}^{1}, q_{j}^{2}\right)\right)$ :

$\mathcal{E}_{1}=$ fewer than a $1 / 8$-fraction of the vertices $v \in C_{i}^{1}$ satisfy $f(v) \neq f(\bar{v})$.

$\mathcal{E}_{2}=M$ contains more than $2^{d+5} L$ hypercube edges in $C_{i}^{1}$.

$\mathcal{E}_{3}=M$ contains more than $2^{d+5} L$ hypercube edges in $C_{j}^{2}$.

$\mathcal{E}_{4}=M$ contains more than $2^{d+5} \eta L$ cross edges between $C_{i}^{1}$ and $C_{j}^{2}$.

First, by our assumption above, removing $M$ disconnects at least an $\alpha \geq 7 / 8$ fraction of the demand pairs inside the cubes $\left\{C_{l}^{1}\right\}_{l \in[n]}$. Thus, the expected fraction of demand pairs $(v, \bar{v})$ in $C_{i}^{1}$ that are not disconnected in $G \backslash M$ (and thus clearly $f(v)=f(\bar{v})$ is at most $1 / 8$. In addition, the expected fraction of demand pairs $(v, \bar{v})$ in $C_{i}^{1}$ that are disconnected in $G \backslash M$ and satisfy $f(v)=$ $f(\bar{v})$ is at most $1 / 2$, because different connected components of $G \backslash M$ are labeled independently. Thus, the expected fraction of vertices $v \in C_{i}^{1}$ for which $f(v)=f(\bar{v})$ is at most 5/8, and by Markov's inequality, $\operatorname{Pr}\left[\mathcal{E}_{1}\right] \leq 5 / 7$. Next, the cutset $M$ contains at most $2^{d+1} n L$ hypercube edges, thus the expected number of edges in $C_{i}^{1} \cup C_{j}^{2}$ that are contained in $M$ is at most $2^{d+1} L$, and by Markov's inequality $\operatorname{Pr}\left[\mathcal{E}_{2} \cup \mathcal{E}_{3}\right] \leq 1 / 16$. Finally, $\operatorname{Pr}\left[\mathcal{E}_{4}\right] \leq 1 / 16$, as otherwise the total cost of $M$ along the cross edges corresponding to this event is more than $1 / 16 \cdot\left(2^{d+5} \eta L\right) \cdot \Lambda=2^{d+1} n L \geq c(M)$. Taking a union bound, we upper bound the probability that any of the bad events occurs by

$$
\operatorname{Pr}\left[\mathcal{E}_{1} \cup \mathcal{E}_{2} \cup \mathcal{E}_{3} \cup \mathcal{E}_{4}\right] \leq \frac{5}{7}+\frac{2}{16}<\frac{6}{7} .
$$

In order to lower bound the expected value of the randomized solution $A$, we would like to show that if none of the four bad events above happens, then the two sets of dimensions $J_{i}^{1}$ and $J_{j}^{2}$ obtained using Friedgut's Junta Theorem are relatively small, and further they are in "weak agreement", and these two properties will immediately imply that the randomized solution $A$ satisfies $\operatorname{Pr}\left[b_{i j}\left(A_{i}^{1}\right)=A_{j}^{2}\right]>\delta$. Observe that if $(u, v) \in E$ and $f(u) \neq f(v)$, then $(u, v) \in M$. If the event $\mathcal{E}_{2}$ does not occur, then the total influence of $f_{\mid C_{i}^{1}}$ is at most $64 L$, and thus $\left|J_{i}^{1}\right| \leq 2^{O(L / \varepsilon)}$. Similarly, if the event $\mathcal{E}_{3}$ does not occur, then the total influence of $f_{\mid C_{j}^{2}}$ is at most $64 L$, and thus $\left|J_{j}^{2}\right| \leq 2^{O(L / \varepsilon)}$. In addition, if the event $\mathcal{E}_{1}$ does not occur, then the balance of $f_{\mid C_{i}^{1}}$ is at least $1 / 16$. 
We now claim that if none of the above bad events happens then there is $a \in J_{i}^{1}$ for which $b_{i j}(a) \in J_{j}^{2}$. Indeed, assume towards a contradiction that $J_{i}^{1} \cap b_{i j}^{-1}\left(J_{j}^{2}\right)=\emptyset$. Then by construction there is a Boolean function $g_{i}^{1}: C_{i}^{1} \rightarrow$ $\{0,1\}$ that is $\varepsilon$-close to $f_{\mid C_{i}^{1}}$ and depends on variables in $J_{i}^{1}$ only. Clearly, the balance of $g_{i}^{1}$ is close to that of $f_{\mid C_{i}^{1}}$, namely, at least $1 / 16-\varepsilon$. Similarly, there is a Boolean function $g_{j}^{2}: C_{j}^{2} \rightarrow\{0,1\}$ that is $\varepsilon$-close to $f_{\mid C_{j}^{2}}$ and depends on variables in $J_{j}^{2}$ only. We can relate these two functions via $b_{i j}^{\prime}: C_{i}^{1} \rightarrow C_{j}^{2}$, namely by considering $h: C_{i}^{1} \rightarrow\{0,1\}$ given by $h(v)=g_{j}^{2}\left(b_{i j}^{\prime}(v)\right)$.

Notice that $h$ is $\varepsilon$-close to $f_{\mid C_{j}^{2}} \circ b_{i j}^{\prime}$, and that it depends only on variables in $b_{i j}^{-1}\left(J_{j}^{2}\right)$. Therefore $g_{i}^{1}$ and $h$ depend on disjoint sets of variables. It follows that $\operatorname{Pr}_{v \in C_{i}^{1}}\left[g_{i}^{1}(v) \neq h(v)\right] \geq 1 / 16-\varepsilon$, because if we condition on the value of the variables in $b_{i j}^{-1}\left(J_{j}^{2}\right)$ we see that $h(v)$ is determined, but this does not affect the distribution of $g_{i}^{1}(v)$, which still attains each value ( 0 or 1$)$ with probability at least $1 / 16-\varepsilon$. Consequently, $g_{i}^{1}$ and $h=g_{j}^{2} \circ b_{i j}^{\prime}$ are not, say, $(1 / 17-\varepsilon)$-close.

On the other hand, the event $\mathcal{E}_{4}$ not occurring implies that at most $32 \eta L 2^{d}$ vertices $v \in C_{i}^{1}$ satisfy $f(v) \neq f\left(b_{i j}^{\prime}(v)\right)$. In other words, $f_{\mid C_{i}^{1}}$ is $(32 \eta L)$-close to $f_{\mid C_{j}^{2}} \circ b_{i j}^{\prime}$. The former is $\varepsilon$-close to $g_{i}^{1}$ while the latter is $\varepsilon$-close to $g_{j}^{2} \circ b_{i j}^{\prime}$ (because $b_{i j}^{\prime}$ is a bijection on the variables), and by the triangle inequality we find that $g_{i}^{1}$ and $h=g_{j}^{2} \circ b_{i j}^{\prime}$ are $(2 \varepsilon+32 \eta L)$-close. If $c>0$ is sufficiently small, $2 \varepsilon+32 \eta L<1 / 17-\varepsilon$, which yields a contradiction and completes the proof of the claim.

Using the above claim we infer that for a random question edge,

$$
\begin{aligned}
\operatorname{Pr}\left[A_{j}^{2}=b_{i j}\left(A_{i}^{1}\right)\right] & \geq \operatorname{Pr}\left[A_{i}^{1} \in J_{i}^{1} \cap b_{i j}^{-1}\left(J_{j}^{2}\right), A_{j}^{2}=b_{i j}\left(A_{i}^{1}\right)\right] \\
& \geq \frac{1}{7} \cdot 2^{-O(L / \varepsilon)} \cdot 2^{-O(L / \varepsilon)}=2^{-O(L)} .
\end{aligned}
$$

We conclude that the expected value of the randomized solution $A$ is given by $\operatorname{Pr}\left[A_{j}^{2}=b_{i j}\left(A_{i}^{1}\right)\right] \geq 2^{-O(L)}>\delta$, where the last inequality holds if $c>0$ is sufficiently small, and this completes the proof of Lemma 2.3 .

2.5. Putting it all together. The above reduction from unique 2-prover game to Multicut produces a gap of $L(n)=\Omega(\min \{1 / \eta(n), \log (1 / \delta(n))\})$. We assumed $d(\eta, \delta) \leq O(\log n)$, and thus the resulting Multicut instance $G$ has size $N=\left(n 2^{d}\right)^{O(1)}=n^{\Theta(1)}$. It follows that in terms of the Multicut instance size $N$, the gap is

$$
L(N)=\Omega\left(\min \left\{\frac{1}{\eta\left(N^{\Theta(1)}\right)}, \log \frac{1}{\delta\left(N^{\Theta(1)}\right)}\right\}\right) .
$$


This completes the proof of the part of Theorem 1.2 regarding the MULTiCuT problem, namely, that the Unique Games Conjecture implies that it is NPhard to approximate Multicut within the above factor $L(N)$. In fact, the above proof shows that it is NP-hard to obtain even a $(7 / 8, L(N))$-bicriteria approximation.

Note that the number of demand pairs is $k=n 2^{d}=n^{\Theta(1)}$, and thus the hardness of approximation factor is similar when expressed in terms of $k$ as well. Note also that all edge weights in the MuLTicut instance constructed above are bounded by a polynomial in the size of the graph. Therefore, via a standard reduction, a similar hardness result holds for the unweighted MuLticuT problem as well.

\section{Hardness of approximating SpARSEST-CuT}

In this section we prove the part of Theorem 1.2 regarding the SPARSEST-CUT problem. The proof follows immediately from the next lemma in conjunction with the hardness of bicriteria approximation of MulTicut (from Section 2).

Lemma 3.1. Let $0<\alpha<1$ be a constant. If there exists a polynomial-time algorithm for SPARSEST-CUT that produces a cut whose value is within factor $\rho \geq 1$ of the minimum, then there is a polynomial time algorithm that computes an $\left(\alpha, \frac{\rho}{1-\alpha}\right)$-bicriteria approximation for Multicut.

Proof. Fix $0<\alpha<1$, and suppose $\mathcal{A}$ is a polynomial-time algorithm for Sparsest-Cut that produces a cut whose value is within factor $\rho \geq 1$ of the minimum. Now suppose we are given an input graph $G=(V, E)$ and $k$ demand pairs $D=\left\{\left\{s_{1}, t_{1}\right\}, \ldots,\left\{s_{k}, t_{k}\right\}\right\}$. We may assume without loss of generality that every $s_{i}$ is connected (in $G$ ) to its corresponding $t_{i}$.

We now describe the bicriteria approximation algorithm for Multicut. It is an iterative algorithm where $M=\emptyset$ at the beginning and the following is executed at each iteration: we apply algorithm $\mathcal{A}$ to $G$ with $D$ as the set of demand pairs, letting $M^{\prime}$ be the cutset produced by $\mathcal{A}$ and $D^{\prime}$ be the demand pairs in $D$ that are disconnected by $M^{\prime}$, and then set $M \leftarrow M \cup M^{\prime}$ and $D \leftarrow D \backslash D^{\prime}$. Note that $D$ changes throughout the iterations, but $G$ does not. The iterations proceed as long as $|D| \geq(1-\alpha) k$, and eventually the final $M$ is output.

This algorithm clearly runs in polynomial time, and there are at most $\alpha k$ iterations. For the sake of analysis, fix an optimal multicut $M^{*} \subseteq E$, i.e., a cutset of $G$ whose removal disconnects all the demand pairs and has the least cost. We first claim that in every iteration, there is a cut whose value (in the 
SpARSEST-CUT sense) is at most $c\left(M^{*}\right) /(1-\alpha) k$; this follows since the number of remaining demands pairs is $|D| \geq(1-\alpha) k$ and removing $M^{*}$ disconnects all these demand pairs. By the approximation guarantees of $\mathcal{A}$, at every iteration we have

$$
c\left(M^{\prime}\right) \leq \rho \cdot \frac{c\left(M^{*}\right)}{(1-\alpha) k} \cdot\left|D^{\prime}\right| .
$$

Summing over all the iterations, we can upper bound the cost of the output cut by

$$
c(M) \leq \rho \cdot \frac{c\left(M^{*}\right)}{(1-\alpha) k} \cdot k=\frac{\rho}{1-\alpha} c\left(M^{*}\right)
$$

Clearly, the output cut disconnects at least $\alpha k$ demand pairs in $G$, and this concludes the proof of the lemma.

\section{Hardness of approximating MIN-2CNF $\equiv$ DELETION}

In this section, we modify the reduction in Section 2.1 to obtain a hardness of approximation for MIN-2CNF $\equiv$ DELETION. In particular, we reduce the Multicut instance obtained in Section 2.1 to Min-2CNF $\equiv$ Deletion, such that a solution to the latter gives a Multicut of the same cost in the former.

The Min-2CNF $\equiv$ DELETION instance contains $2^{d} n$ variables, one for each demand pair $\{u, \bar{u}\}$. In particular, for every demand pair $\{u, \bar{u}\} \in D$, we associate the literal $x_{u}$ with $u$ and the literal $x_{\bar{u}}=\neg x_{u}$ with $\bar{u}$. For every edge $e=(u, v)$ in the graph $G$ there is a clause $\left(x_{u} \equiv x_{v}\right)$ whose weight is equal to the edge-weight $w_{e}$.

The following lemma is immediate from the construction and implies an analog of Lemma 2.3 for MiN-2CNF $\equiv$ DELETION.

Lemma 4.1. Given an assignment $S$ of $\operatorname{cost} W$ to the above instance of Min$2 \mathrm{CNF} \equiv$ DeLETION, we can construct a solution of cost $W$ to the Multicut instance $G$.

Proof. Let $M$ be the set of edges $(u, v)$ for which $S\left(x_{u}\right) \neq S\left(x_{v}\right)$. Then $M$ corresponds to the clauses that are not satisfied by $S$ and has weight $W$. The lemma follows from observing that $M$ is indeed a multicut $-S$ is constant over connected components in $G \backslash M$, and for every demand pair $(u, \bar{u})$, we have $S\left(x_{u}\right) \neq S\left(x_{\bar{u}}\right)$.

We now give an analog of Lemma 2.1. 
Lemma 4.2. If there is a solution $A$ for the unique 2-prover game $G_{Q}$ such that the total weight of the satisfied questions is at least $1-\eta$, then there exists an assignment $S$ for the above Min-2CNF $\equiv$ DeLetion instance such that $c(S) \leq 2^{d+1} n$.

Proof. Given the solution $A$ for $G_{Q}$, we construct an assignment $S$ as follows. For every question $q_{i}^{p}$ and for every vertex $u$ in the corresponding hypercube $C_{i}^{p}$, define $S\left(x_{u}\right)$ to be the $A_{i}^{p}$-th bit of $u$, i.e., $S\left(x_{u}\right)=u_{A_{i}^{p}}$. Note that this is a valid assignment, i.e., $S\left(x_{u}\right)=1-S\left(x_{\bar{u}}\right)$ for all vertices $u$, as $u_{A_{i}^{p}}=1-\bar{u}_{A_{i}^{p}}$.

We bound the cost of the solution by first analyzing the clauses corresponding to hypercube edges in the corresponding Multicut instance. Consider unsatisfied clauses containing both variables in the same hypercube $C_{i}^{p}$, and note that the hypercube edges corresponding to these clauses form a dimension$A_{i}^{p}$ cut in the cube $C_{i}^{p}$. Therefore, the total weight of these clauses is at most $\left(2^{d-1}\right)(2 n)=2^{d} n$.

Finally, consider an unsatisfied clause $\left(x_{u} \equiv x_{v}\right)$ corresponding to vertices in different hypercubes $C_{i}^{1}$ and $C_{j}^{2}$. Then $S\left(x_{u}\right) \neq S\left(x_{v}\right)$ implies that $u_{A_{i}^{1}}=$ $v_{b_{i j}\left(A_{i}^{1}\right)} \neq v_{A_{j}^{2}}$, or $b_{i j}\left(A_{i}^{1}\right) \neq A_{j}^{2}$. There are at most $2^{d}$ such clauses for each question pair not satisfied by the solution $A$. Therefore, the total weight of such clauses is at most $2^{d} \eta \Lambda=2^{d} n$.

The lemma follows from adding the total weight of these two types of edges.

Lemmas 4.1 and 4.2 along with Lemma 2.3 imply the part of Theorem 1.2 regarding Min-2CNF $\equiv$ DELETION.

\section{Concluding remarks}

Several important questions are left open. First, one would like to eliminate the dependence on the Unique Games Conjecture, and obtain a "standard" hardness of approximation result. Yet another challenge is to improve the hardness factor. For Multicut, the $\Omega(\log k)$ integrality ratio lower bound of Agarwal et al. (2005) suggests that the inapproximability bound may be improved. In particular, $(\log k)^{c}$ hardness for a constant $c>1 / 2$ will separate the approximability of MULTiCUT from that of SPARSEST-CUT (in light of the recent approximation due to Arora et al. 2005). The main bottleneck to improving the hardness factor lies in Friedgut's Junta Theorem (and similarly in the result of Kahn et al. (1988) that we used in the conference version). These bounds are tight in general, as shown by the tribes function (Ben-Or \& Linial 1989) (see also Friedgut 1998, Section 2). 
A third challenge is to obtain hardness of approximation results for the uniform-demand case of the SpARSEST-CuT problem or for the BALANCEDCut problem. Our results do not apply to this special but important case; in particular, if a 2-prover system has a low-cost balanced cut, then the corresponding graph on hypercubes would have a low-cost balanced cut regardless of the value of the 2-prover game. Very recently, Devanur et al. (2006) have shown a lower bound of $\Omega(\log \log n)$ on the integrality ratio of the natural SDP relaxation for this problem. Alternatively, of course, one might improve the approximation algorithms for any of these problems.

\section{Acknowledgements}

We thank the anonymous referees for useful comments, and in particular for suggesting a simplification of our original proof of Lemma 3.1. Part of this work was done while Shuchi Chawla was visiting IBM Almaden Research Center. Part of this work was done while Yuval Rabani was on sabbatical leave at Cornell University, while visiting IBM Almaden Research Center, and while visiting the Institute for Pure and Applied Mathematics at UCLA; his research at the Technion was supported in part by ISF grant number 52/03 and BSF grant number 02-00282. This work was done while Ravi Kumar and D. Sivakumar were at the IBM Almaden Research Center. A preliminary version of this paper appeared in Proceedings of 20th Annual IEEE Conference on Computational Complexity (CCC 2005).

\section{References}

A. Agarwal, M. Charikar, K. Makarychev \& Y. Makarychev (2005). $O(\sqrt{\log n})$ approximation algorithms for Min UnCut, Min 2CNF Deletion, and directed cut problems. In Proc. 37th Annual ACM Symposium on Theory of Computing, $573-581$.

S. Arora, J. R. Lee \& A. Naor (2005). Euclidean distortion and the sparsest cut. In Proc. 37th Annual ACM Symposium on Theory of Computing, 553-562.

S. Arora, C. Lund, R. Motwani, M. Sudan \& M. Szegedy (1998). Proof verification and the hardness of approximation problems. J. ACM 45, 501-555.

S. Arora, S. Rao \& U. Vazirani (2004). Expander flows, geometric embeddings, and graph partitionings. In Proc. 36th Annual ACM Symposium on Theory of Computing, 222-231. 
S. Arora \& S. Safra (1998). Probabilistic checking of proofs: A new characterization of NP. J. ACM 45, 70-122.

Y. Aumann \& Y. Rabani (1998). An $O(\log k)$ approximate min-cut max-flow theorem and approximation algorithm. SIAM J. Comput. 27, 291-301.

N. Bansal, A. Blum \& S. Chawla (2004). Correlation clustering. Machine Learning 56, Special Issue on Clustering, 89-113.

M. Bellare, O. Goldreich \& M. Sudan (1998). Free bits, PCP's and nonapproximability - towards tight results. SIAM J. Comput. 27, 804-915.

M. Ben-Or \& N. Linial (1989). Collective coin flipping. In Randomness and Computation, S. Micali (ed.), JAI Press, 91-115.

M. Charikar, V. Guruswami \& A. Wirth (2003). Clustering with qualitative information. In Proc. 44th IEEE Symposium on Foundations of Computer Science, $524-533$.

M. Charikar, K. Makarychev \& Y. Makarychev (2006). Near-optimal algorithms for Unique Games. In Proc. 38th Annual ACM Symposium on Theory of Computing, to appear.

S. Chawla, A. Gupta \& H. RÄCKe (2005a). Improved approximations to sparsest cut. In Proc. 16th Annual ACM-SIAM Symposium on Discrete Algorithms, 102-111.

S. Chawla, R. Krauthgamer, R. Kumar, Y. Rabani \& D. Sivakumar (2005b). On the hardness of approximating multicut and sparsest-cut. In Proc. 20th Annual IEEE Conference on Computational Complexity, 144-153.

E. Dahlhaus, D. S. Johnson, C. H. Papadimitriou, P. D. Seymour \& M. YanNAKAKIS (1994). The complexity of multiterminal cuts. SIAM J. Comput. 23, 864894.

E. Demaine \& N. Immorlica (2003). Correlation clustering with partial information. In Proc. 6th International Workshop on Approximation Algorithms for Combinatorial Optimization Problems (APPROX), 1-13.

N. Devanur, S. Khot, R. Saket \& N. Vishnoi (2006). Integrality gaps for sparsest cut and minimum linear arrangement problems. In Proc. 38th Annual ACM Symposium on Theory of Computing, to appear.

D. Emanuel \& A. Fiat (2003). Correlation clustering - minimizing disagreements on arbitrary weighted graphs. In Proc. 11th Annual European Symposium on Algorithms, 208-220. 
U. Feige \& D. Reichman (2004). On systems of linear equations with two variables per equation. In Proc. 7th International Workshop on Approximation Algorithms for Combinatorial Optimization Problems (APPROX), 117-127.

E. Friedgut (1998). Boolean functions with low average sensitivity depend on few coordinates. Combinatorica 18, 27-35.

N. Garg, V. V. Vazirani \& M. Yannakakis (1996). Approximate max-flow min-(multi)cut theorems and their applications. SIAM J. Comput. 25, 235-251.

A. Gupta \& K. Talwar (2006). Approximating unique games. In Proc. 17th Annual ACM-SIAM Symposium on Discrete Algorithms, 99-106.

J. HÅstad (2001). Some optimal inapproximability results. J. ACM 48, 798-859.

J. Kahn, G. Kalai \& N. Linial (1988). The influence of variables on Boolean functions. In Proc. 29th IEEE Symposium on Foundations of Computer Science, $68-80$.

S. Кнот (2002). On the power of unique 2-prover 1-round games. In Proc. 34th Annual ACM Symposium on Theory of Computing, 767-775.

S. Khot, G. Kindler, E. Mossel \& R. O'Donnell (2004). Optimal inapproximability results for MAX-CUT and other 2-variable CSPs. In Proc. 45th IEEE Symposium on Foundations of Computer Science, 146-154. See also ECCC Report TR05-101, 2005.

S. Khot \& O. Regev (2003). Vertex cover might be hard to approximate to within 2- $\epsilon$. In Proc. 18th Annual IEEE Conference on Computational Complexity, 379-386.

S. Кнот \& N. K. Vishnoi (2005). The unique games conjecture, integrality gap for cut problems and the embeddability of negative type metrics into $\ell_{1}$. In Proc. 46th IEEE Symposium on Foundations of Computer Science, 53-62.

P. Klein, A. Agrawal, R. Ravi \& S. Rao (1990). Approximation through multicommodity flow. In Proc. 31st IEEE Symposium on Foundations of Computer Science, 726-737.

R. Krauthaamer \& Y. Rabani (2006). Improved lower bounds for embeddings into $L_{1}$. In Proc. 17th Annual ACM-SIAM Symposium on Discrete Algorithms, ACM, 1010-1017.

F. T. Leighton \& S. RaO (1988). An approximate max-flow min-cut theorem for uniform multicommodity flow problems with applications to approximation algorithms. In Proc. 29th IEEE Symposium on Foundations of Computer Science, 422-431. 
N. Linial, E. London \& Y. Rabinovich (1995). The geometry of graphs and some of its algorithmic applications. Combinatorica 15, 215-245.

E. Mossel, R. O'Donnell \& K. Oleszkiewicz (2005). Noise stability of functions with low influences: Invariance and optimality. In Proc. 46th IEEE Symposium on Foundations of Computer Science, 21-30.

R. RAZ (1998). A parallel repetition theorem. SIAM J. Comput. 27, 763-803.

L. Trevisan (2005). Approximation algorithms for unique games. In Proc. 46th IEEE Symposium on Foundations of Computer Science, 197-205. See also comment for ECCC TR05-34.

V. V. Vazirani (2001). Approximation Algorithms. Springer, Berlin.

Manuscript received 19 September 2005

\author{
Shuchi ChaWla \\ Computer Science Department \\ Carnegie Mellon University \\ Pittsburgh, PA 15213, U.S.A. \\ shuchi@cs.cmu.edu \\ RAVI KUMAR \\ IBM Almaden Research Center \\ 650 Harry Road \\ San Jose, CA 95120, U.S.A. \\ Yuval RABANi \\ Computer Science Department \\ Technion-Israel Institute of Technology \\ Haifa 32000, Israel \\ rabani@cs.technion.ac.il \\ Current address of D. SivakumaR: \\ Google, Inc. \\ 1600 Amphitheater Parkway \\ Mountain View, CA 94043, U.S.A. \\ siva@google.com
}

ROBERT KRAUTHGAMER

IBM Almaden Research Center

650 Harry Road

San Jose, CA 95120, U.S.A.

robi@almaden.ibm.com

Current address of RAvi KumaR:

Yahoo! Research

701 First Avenue

Sunnyvale, CA 94089, U.S.A.

ravikumar@yahoo-inc.com

D. SIVAKUMAR

IBM Almaden Research Center

650 Harry Road

San Jose, CA 95120, U.S.A. 